\title{
"LUCHAR" POR TRABAJO, TRABAJAR "LUCHANDO": PRÁCTICAS COTIDIANAS DE ORGANIZACIÓN Y DEMANDA EN UNA EMPRESA RECUPERADA DE BUENOS AIRES.
}

\author{
María Inés FERNÁNDEZ ÁLVAREZ ${ }^{1}$
}

\section{Resumen}

El artículo retoma resultados de una investigación etnográfica sobre empresas recuperadas de la Ciudad de Buenos Aires entre 2002 y 2005. Analizo las prácticas cotidianas desarrolladas por las/los trabajadoras/es mostrando los cambios en la jornada de trabajo a partir de los cuales el "lugar de trabajo" se convirtió en un "espacio de lucha" y en sentido inverso el "espacio de lucha" fue organizado como un "lugar de trabajo". Sugiero que esta articulación entre "lucha" y "trabajo" permitió la construcción de un innovador proceso de demanda desarrollando desde la práctica cotidiana modos renovados de hacer política y trabajo.

Palabras Claves: empresas recuperadas; trabajo; política; practicas cotidianas; etnografía

\section{Abstract}

The present paper summarizes my ethnograpic studies on recovered factories in Buenos Aires which took place between 2002 and 2005. I analyse the everyday practices of workers showing changes in the working day. As a result of these changes the "workplace" became a "site of struggle" and, at the same time, the "site of struggle" was defined as a "workplace". I suggest that this articulation of "work" and "struggle" allowed the building up of an innovative process of claims, thus developing new ways of doing politics and labor based on the everyday practices.

Key Wods: Recovered factories; work; politic; every day practices; ethnography

\section{Resumé}

Cet article reprends les résultat d'une recherche ethnographique sur les usines récuperées dans la Ville de Buenos Aires entre 2002 et 2005. J'analyse les pratiques quotidiennes des travailleuses/eurs mettant en évidence les changements dans la journé de travail. Comme

\footnotetext{
${ }^{1}$ Investigadora Asistente CONICET - Docente regular Carrera de Ciencias Antropológicas, Facultad de Filosofía y Letras, Universidad de Buenos Aires.

mifernandezalvarez@gmail.com; mfernandezalvarez@conicet.gov.ar
}

Fecha de recepción del artículo: Abril 2012

Fecha de evaluación:Mayo 2012 
conséquence de ces changements le «lieu de travail» est devenue un «espace de lutte» et au même temps «l'espace de lutte» est organisé comme un «lieu de travail». Je soutiens que cette articulation entre «lutte» et «travail» a permis la construction d'un processus innovateur de demande et le développement a partir leur quotidienne des practiques renouve de politique et travail.

Mots clé: usines récuperées; travail; politique; pratiques quotidiennes; ethnographie

\section{Introducción}

En abril del año 2002 tomé contacto con trabajadoras/es de una fábrica de confecciones recientemente ocupada y puesta a producir bajo gestión obrera. Una mujer de unos 50 años vestida con un guardapolvo celeste me invitó a pasar. Con suma ingenuidad intenté explicarle de la manera más clara posible el propósito de mi visita. "Soy egresada de antropología de la UBA, estoy haciendo un proyecto de investigación sobre las transformaciones en el mundo del trabajo en Argentina y me gustaría acercarme a la fábrica"2, le dije según lo dejé asentado en mi cuaderno de campo. Sin sorprenderse demasiado por mi presentación me informó que tenía que hablar con la "encargada de prensa" a quien llamó por el interno. Quince minutos después me encontraba charlando con Clara una mujer muy amable que como me contaría tiempo después llevaba 28 años trabajando en el oficio y más de diez en la fábrica. Concluida mi presentación -que sumó a la versión previa algunas informaciones sobre el propósito de mi proyecto del que le entregué una copia impresa- Clara relató muy brevemente los acontecimientos trascurridos desde el momento en que habían iniciado la ocupación, explicándome que tras varios meses de sueldos atrasados los dueños habían "abandonado la fábrica" y ellas/os reclamaban el mantenimiento de "la fuente de trabajo". Mostrándose dispuesta a recibirme, cerró la charla diciéndome: "lo mejor sería que vuelvas después de las $15 \mathrm{hs}$, cuando estemos fuera del horario de trabajo".

Cumpliendo con lo acordado, en las semanas siguientes visité la fábrica de manera regular después de las tres de la tarde. En contraste con la sensación de tranquilidad que me había llevado esa mañana, el ambiente de las visitas posteriores estuvo marcado por una permanente circulación de personas que se acercaban a la fábrica con propósitos variados: comprar un producto, hacer una nota periodística, realizar un estudio sociológico, apoyar "la lucha" o militar. Esa circulación daba cuenta de la amplia notoriedad pública que la fábrica había cobrado constituyéndose en uno de los casos más emblemáticos dentro de las empresas recuperadas ${ }^{4}$. Durante esas primeras visitas percibí que el día a día en la fábrica se organizaba

\footnotetext{
${ }^{2}$ Utilizo comillas dobles para citas textuales, bastardillas para categorías nativas y comillas simples para relativizar o destacar términos. Los nombres propios han sido modificados en función de garantizar la confidencialidad de los datos.

3 Las narraciones sobre el inició de la ocupación explican que esta comenzó como resultado del incumplimiento del pago de salario y el posterior abandono de la empresa por parte de la patronal. Según este relato a partir del año 1998 los dueños habían suspendido los aportes a la seguridad social y desde el 2000 habían interrumpido el pago del sueldo quincenal reemplazado por un vale semanal que consistía en un adelanto de la quincena cuyo monto fue decayendo durante el 2001 para alcanzar en la última semana la suma de 5 pesos. Este relato se completaba con la descripción de escenas como el retiro de pertenencias personales de los dueños o maquinas del establecimiento y el anuncio de vacaciones para el conjunto de las/os empleados que denotaban un posible proceso de "vaciamiento" y cierre de la planta.

${ }^{4}$ Con esta denominación se hizo referencia a una forma específica de demandar por la "fuente de trabajo" que incluyó en la amplia mayoría de los casos la ocupación del inmueble, la gestión colectiva de la producción, el desarrollo de negociaciones con diferentes agencias del estado para lograr la continuidad de la unidad productiva y la conformación de una cooperativa de trabajo. Como lo he desarrollado en trabajos previos (Fernández Álvarez, 2007), si bien las empresas recuperadas se multiplicaron y cobraron notoria visibilidad a partir de los acontecimientos de diciembre de 2001 esos procesos venían
} 
entre el "horario laboral" y el tiempo "de lucha". Aunque en los meses siguientes fui comprendiendo que esta era una distinción cuyos límites se hacían bastante más difusos, "lucha" y "trabajo" eran dos palabras que se conjugaban en tensión y explicaban los ritmos, prácticas, lenguajes y relaciones que allí se tramaban. En síntesis, en esos primeros meses fui descubriendo que el universo en el que estaba ingresando iba mucho más allá "del mundo del trabajo". O más precisamente, aunque el trabajo constituía un eje reivindicativo era en su articulación con categorías como "lucha" o "dignidad" que cobraba pleno sentido.

Como parte de un escenario más amplio de movilización social del que era objeto la Argentina a comienzos del nuevo siglo, las empresas recuperadas rápidamente despertaron una fuerte atracción por parte de académicas/os, militantes e intelectuales que encontraron en estas experiencias la expresión de una respuesta al neoliberalismo o la globalización (Magnani, 2003; Palomino, 2003; Rofman y otros, 2003; Petras y Vetmeyer, 2002; Davalos y Perelman, 2005). Emparentadas con otros procesos desarrollados en la región como las ocupaciones de tierra en Brasil, la guerra del agua en Bolivia o las reivindicaciones por la autonomía de los zapatistas en México, las empresas recuperadas se sumaron a la construcción de visiones alternativas al neoliberalismo en respuesta al avance de los procesos de globalización sobre modos locales de vida (Nash, 2005; 2006). A nivel local, esta discusión se inscribió en un debate más amplio sobre las formas de movilización social y política desarrolladas en Argentina post-2001 que buscaron explicar la emergencia de nuevos actores y repertorios de acción. En esta línea, algunas investigaciones atendieron al carácter innovador de estos procesos como formas de protesta o acción colectiva (Fajn, 2003; Favaro y Aizicson, 2003; Gracia y Cavaliere; 2007; Davalos y Perelman, 2005) $)^{5}$ Estudios posteriores, ampliaron la pregunta al ámbito de la producción aportando al estudio sobre la organización del trabajo (Rebon y Fajn, 2005; Hudson, 2007; Rebon y Salgado, 2009) o recuperaron aportes de la economía política para inscribir este fenómeno en un análisis de la relación capital-trabajo (Trinchero, 2007; Ruggeri, 2009). De esta manera, si el primer conjunto de trabajos tuvo como eje de indagación la 'dimensión política' de las recuperadas, el segundo conjunto focalizó en el trabajo indagando sobre las potencialidades y los límites que la forma cooperativa o autogestiva alcanzaba en el marco de relaciones capitalistas de producción.

El propósito de este artículo es contribuir a este campo de estudios introduciendo un desplazamiento que consiste en considerar ambos aspectos, la política y el trabajo, en su articulación. Retomo para ello resultados de un estudio etnográfico sobre empresas recuperadas de la Ciudad de Buenos Aires que realicé entre 2002 y 2005 -período en el que se concentró el número más elevado de casos-. En el marco de este proceso el espacio "de trabajo" se convirtió también en un espacio "de lucha" redefiniendo los tiempos y actividades comprendidas en la "jornada laboral". En sentido inverso el espacio "de lucha" (la realización de movilizaciones, acampes, cortes de calle, etc.) fue definido como un "lugar de trabajo", regulado, organizado y

desarrollándose desde mediados de los años noventa con antecedentes directos en la década del 80 . Según diferentes estudios (Rebon, 2007; Ruggieri, 2009), en nuestro país se han desarrollado aproximadamente unas 200 recuperaciones de fábricas que alcanzan 10000 trabajadoras/es en todo el país, de los cuales el $60 \%$ tuvo lugar en la región de Buenos Aires. En particular en el caso de la Ciudad de Buenos Aires -al que circunscribí mi estudio- para el año 2009 el número total de recuperadas alcanzaba 41 casos y 2066 trabajadoras/es, $68 \%$ de los cuales comenzó en 2002. La mayoría de las empresas recuperadas pertenecen al segmeno de pequeñas y medianas empresas (es decir, aquellas que no cuentan con más de 50 empleadas/os), aunque la existencia de unidades de mayor tamaño no deja de ser significativa. Las actividades productivas se distribuyen mayoritariamente en la rama industrial -principalmente metalúrgicas, frigoríficas, textiles, del plástico o gráficas- y en menor medida en servicios.

5 En discusión con estas miradas otros estudios analizaron el fenómeno de las empresas recuperadas desde perspectivas marxistas partiendo de la noción de clase (Martínez, 2002; Petras y Veltmeyer; 2002; Rebon, 2007). En particular el trabajo de J. Rebon (2007) ha propuesto una mirada que anclando en esta tradición y analizando el carácter de clase de las relaciones sociales recupera aportes de otras corrientes teóricas contribuyendo a explicar la socio-génesis de estos procesos. 
significado como tal. A partir de la reconstrucción de un caso en particular, este artículo analiza el modo en que las actividades productivas se articularon en acciones de protesta o, más precisamente la recuperación tomó la forma de una acción política anclada en la producción. Con este objetivo, en la primera parte del artículo reconstruyo la vida cotidiana en una fábrica recuperada mostrando cómo la "jornada de trabajo" desbordó los límites de "lo productivo". En un segundo momento analizó el proceso de trabajo focalizando en uno de los principales cambios introducidos a partir de la recuperación: la rotación de los puestos de trabajo. Finalmente, abordo un momento específico del proceso de demanda en función de analizar cómo las prácticas de protesta cobraron ritmos, formatos y lenguajes vinculados al mundo de "la producción".

\section{El día a día de una fabrica recuperada: tiempos, ritmos y espacios de "lucha" y "producción".}

A las 8 y media de la mañana se apaga la luz. De manera análoga, como si se tratara de una coreografía delicadamente ensayada, cada uno interrumpe su tarea. El ruido de las máquinas es reemplazado por el de los pasos en busca de mates, yerba, tazas y saquitos de té para la preparación del desayuno. Inmediatamente se encienden las hornallas de una pequeña kichinette instalada en el pasillo contiguo a la sala donde están ubicadas las máquinas. Una mesita cercana reúne a varias trabajadoras que depositan en el centro trozos de pan y bizcochitos. En este espacio destinado al desayuno y al almuerzo desde que "tomaron la producción en sus propias manos", se comparte no sólo aquello que se trajo para comer sino también cuestiones relativas a la organización del trabajo, gestiones referentes a la tramitación de un subsidio o el orden del día de la próxima asamblea. Conversaciones que se entremezclan con el intercambio de recetas, relatos del último fin de semana o secretos para bajar de peso. Del otro lado, algunas sillas se dan vuelta y una de las máquinas cumple la misma función. Dispersos en el fondo hay quienes prefieren, en cambio, permanecer en su lugar de trabajo y aprovechar la pausa para descansar.

Media hora después la luz vuelve a encenderse y las máquinas comienzan a ponerse en funcionamiento. De un lado del salón Carolina limpia $\operatorname{sacos}^{6}$, una tarea que si bien conoce no corresponde al que era su puesto cuando trabaja bajo patrón. A su lado, Lucas su hijo menor, duerme su siesta de la mañana. Muy cerca de ella, en una mesa que está ubicada en el centro del "sector saco" Manuela se ocupa de marcar el delantero y refinar el plastrón?. Si esa era su tarea anteriormente, desde que retomaron la producción bajo gestión obrera intercala esta actividad con otras de administración -algo que aprendió a hacer sobre la marcha a pesar suyo y definitivamente no le gusta- o trámites bancarios -una de sus actividades preferidas porque puede salir un rato de la fábrica-. Rosana, que está ubicada delante de ella, le pasa el delantero cada vez que lo termina mientras da vuelta el cassette de Sandro que antes escuchaba con su walkman y ahora puede sentir a todo volumen entonando cada tanto una frase de la canción. Del otro lado del salón, Julia coloca cinturas ${ }^{8}$ en una máquina que hasta hace algunos meses nunca había usado y que maneja bajo la mirada celosa de Adriana. Desde la recuperación Adriana tuvo que arreglárselas para cambiar de máquina y ceder por momentos "la suya". No es lo único que cambió en su vida. Fue también necesario negociar con su pareja la permanencia en la fábrica algunas noches para cumplir con "las guardias" y aprender a intervenir en las asambleas, un

\footnotetext{
${ }^{6}$ La fábrica en la que centra este artículo se dedica a la confección de prendas de vestir, principalmente trajes de hombres. "Limpiar" significa quitar los hilvanes de la tela una vez que las piezas fueron cocidas.

7 "Refinar" es la acción de cortar los bordes de tela sobrantes de una pieza.

${ }^{8}$ Se llama así a la tarea que consiste en unir las cinturas a los pantalones una vez que ambas piezas fueron confeccionadas por separado.

${ }^{9}$ Una de las primeras acciones realizadas al inicio de la recuperación fue la implementación de una guardia rotativa para garantizar la custodia de la fábrica. Para esto se conformaron grupos estables de seis trabajadoras/es que permanecían en la planta una vez finalizado el horario "de producción", cumpliendo regularmente con un cronograma rotativo de turnos.
} 
espacio en el que con el tiempo comenzó a sentirse cómoda. En el medio un grupo se apresura para agilizar la organización de los lotes de tela que acaban de "bajar" de corte ${ }^{10}$. Varias/os trabajadoras/es interrumpen momentáneamente $s u$ tarea, dándole prioridad a ésta que es ahora más urgente. En un costado, José discute con María la mejor manera de realizar un nuevo pedido, un trabajo a façon ${ }^{11}$ que aunque no es lo que prefieren hacer porque no se trata de "su propia producción" les permite cubrir la quincena y pagar las cuentas. Detrás de ellas/os sobre el pizarrón ubicado en medio del salón se anuncia en letra imprenta: "Viernes 14hs Asamblea". Dolores ya sabe que ese día llegará más tarde de lo habitual a su casa. Deberá prever quien va a buscar al colegio a Andrea, la menor de sus dos hijas.

A las 12 menos diez se corta la luz para dar lugar al almuerzo. Rosana me invita a sentarme con ella en la salita contigua donde come con un grupo de compañeras/os dejando por un momento su máquina. Mientras almorzamos me cuenta que esa tarde tienen que ir a una reunión con otras recuperadas y me invita a acompañarlas/os. La charla gira en torno a la preocupación por un pedido que está atrasado y las discusiones crecientes por el cumplimiento con "la producción". "Hay quienes no terminan de entender que las cosas cambiaron y siguen comportándose como cuando estaban los dueños, en cambio algunas/os creen que pueden ocupar ahora ese lugar. Por eso muchas veces nos peleamos", me aclara Beatriz mientras se levanta a buscar agua. Este intercambio se superpone con una discusión sobre la próxima asamblea en la que se va a votar si interrumpir la producción una hora antes o descontar el día a quienes proponen asistir a la movilización convocada por otra recuperada cuya ley de expropiación se encuentra aún pendiente de aprobación ${ }^{12}$.

\footnotetext{
${ }^{10}$ Se denomina "corte" a la sección donde comienza el proceso de confección de las prendas que consiste en cortar las telas a partir de los moldes correspondientes a cada prenda. Para realizar el corte se utiliza una máquina especial -una cuchilla de cinta accionada por un motor- que se maneja a mano y constituye una de las tareas más calificadas, que según los relatos de las/os trabajadoras/es exige "gran precisión y cuidado, mucho pulso para que no se te safe". En su mayoría es desempeñada por varones.

${ }^{11}$ Este término se utiliza en el ámbito industrial para referir a la manufactura de un producto por encargo de un tercero (dueño de una marca) que suele proveer las materias primas e insumos necesarios para su realización. En este caso lo que se cobra es la mano de obra por la realización de la pieza, lo cual supone una ganancia sumamente inferior a la que se obtiene por la realización del mismo trabajo cuando este es comercializado directamente. Debido a la falta de capital para comprar materias primas, limitaciones en la comercialización de los productos o en la obtención de créditos, muchas empresas recuperadas han tenido que dedicarse (en algunos casos temporalmente) a realizar trabajos bajo esta modalidad. Según un relevamiento del año 2003, el 55\% de los casos se encontraba en esta situación (Fajn, 2003). Este porcentaje parecería tender a reducirse si tomamos en cuenta datos de un informe realizado por el Programa Facultad Abierta (Facultad de Filosofía y Letras, UBA) del año 2010 según el cual el porcentaje de empresas recuperadas que recurre a este tipo de trabajo alcanza el $42 \%$.

La expropiación de las empresas en quiebra constituyó una de las primeras demandas de las empresas recuperadas. En la Ciudad de Buenos Aires se sancionaron y aprobaron 19 leyes de expropiación durante los años 2002 y 2003 que correspondían al 80\% de las empresas recuperadas en esa región. En la mayoría de los casos las leyes declararon de utilidad pública los bienes muebles y patentes de las empresas -casi en su totalidad en situación de quiebra- de manera definitiva, mientras que los bienes inmuebles -a excepción de dos casos- quedaron sujetos a ocupación temporaria por un lapso de dos años. Como hemos analizado en un trabajo previo (Fernández Alvarez, 2007) a diferencia de la demanda de las organizaciones que nucleaban las empresas recuperadas -quienes reclamaron la sanción de una ley nacional de expropiación que afectara al conjunto de las recuperadas-, las leyes se negociaron caso por caso exigiendo a los/las trabajadoras/es la exposición de una serie de pruebas que acreditaran el derecho a esta intervención por parte del Estado. En el año 2004, se decreto la ley 1529 que declaró la expropiación "definitiva" al conjunto de las empresas recuperadas de la Ciudad de Buenos Aires cuyas leyes había sido sancionadas en los años previos, para lo cual el Gobierno de la Ciudad debía pagar las indemnizaciones correspondientes. En noviembre de 2011 el Gobierno Porteño vetó una ley sancionada por la legislatura de dicha Ciudad que prorrogaba por seis años la ley del 2004 cuyas expropiaciones aún no habían sido indemnizadas, dejando a las empresas recuperadas en una situación de extrema fragilidad jurídica.
} 
Treinta minutos después se retoma el trabajo cuyo ritmo se va haciendo cada vez más tranquilo a medida que se acercan las tres de la tarde, momento en que las luces se apagan por última vez anunciando el fin del horario de producción. Mientras algunos emprenden el camino de regreso a casa, Diana, Julia y Rosana se preparan para ir a la reunión con las recuperadas; Inés y Manuela se reúnen en el primer piso para revisar las cuentas de la semana; y Sofía atiende en la planta baja a un cliente que acaba de llegar. Cerca de ella, en la salita contigua a la entrada, Dolores y Roberto dialogan con dos funcionarias del Ministerio de Trabajo sobre la posible entrega de un subsidio para la producción. Horas después, cuando quienes se fueron están llegando a sus casas, nosotras ingresamos a la Cooperativa Vieytes, una recuperada ubicada en el barrio de Barracas. La reunión se extiende hasta pasadas las diecinueve horas. Es demasiado tarde para que Rosana vuelva a su casa. Pasará una vez más la noche en la fábrica.

Con pequeñas variantes, este relato describe a grandes rasgos el día a día en la fábrica ${ }^{13}$. Las actividades cotidianas que realizan en ella las/os trabajadoras/es, no se reducen a la repetición de una operación puntual. Incluyen también tareas relativas a la administración y la venta, negociaciones con funcionarios públicos o reuniones con trabajadoras/es de otras empresas recuperadas. La "jornada de trabajo", que hasta diciembre de 2001 concluía cuando se apagaban las luces, se extendió desde entonces abarcando incluso por momentos los fines de semana ${ }^{14}$. Las actividades que antes correspondían exclusivamente a tareas "de producción" se entremezclaron desde entonces con acciones de "lucha" o "gestión" por la continuidad de la fábrica.

La dinámica del proceso de recuperación, en la que podemos distinguir entre el tiempo "de la lucha" y el tiempo "de la autogestión", imprime ciertos matices a esta rutina: si durante el primero las movilizaciones, actos públicos, encuentros con funcionarios, militantes o dirigentes de otras recuperadas cobraron mayor centralidad; durante el segundo las actividades productivas o de comercialización adquirieron mayor preponderancia. En ambos casos, sin embargo, la recuperación modificó la cotidianeidad de las/os trabajadoras/es, diversificando las actividades desarrolladas y redefiniendo los límites de la jornada y el espacio laboral. Mantener y gestionar la producción se inscribió así en un proceso más amplio por la defensa de "la fuente de trabajo" que combinó actividades productivas con acciones de protesta desdibujando las fronteras entre ambas. Más aun, las segundas quedaron incluidas como parte de la jornada laboral, significadas, reguladas y organizadas como tales. Así, fueron definidas como "responsabilidades" que debían cumplirse al igual que las tareas destinadas estrictamente a la producción. Sin embargo, el modo en que se redefinieron estas fronteras no fue asumido por el conjunto de quienes llevaron adelante la recuperación de manera uniforme. Mientras que para algunas/os el "corte de la luz" siguió marcando el fin de la jornada de trabajo, para otras/os este se modificó completamente prolongándose más allá de las tres de la tarde. En consecuencia, esta diferencia fue generando un espacio cotidiano de tensión y discusión. En función de desplegar esta diferencia, presento a continuación los cambios introducidos en la organización del trabajo.

\footnotetext{
${ }^{13}$ Esta reconstrucción fue elaborada en base a los registros realizados a partir de mis observaciones de campo entre 2002 y 2005. Según mis visitas posteriores (realizadas entre los años 2007 y 2011) esta dinámica cotidiana se mantiene en términos generales.

${ }^{14}$ El proceso de recuperación de esta fábrica comenzó en diciembre de 2001 cuando la planta fue ocupada y los dueños abandonaron la empresa. Durante el mes siguiente se desarrollaron negociaciones en el Ministerio de Trabajo que no lograron resolver el conflicto, mientras las/los trabajadoras/es mantenían la ocupación y demandaban la continuidad de la fuente de trabajo bajo gestión obrera. Entre esa fecha y abril de 2003 se desarrollaron 3 desalojos, el último de los cuales contó con un operativo policial de gran envergadura y finalizó con el vallado de la planta que se mantuvo hasta diciembre de ese mismo año. Durante todo ese tiempo -desde diciembre de 2001 hasta diciembre de 2003- "la custodia de la fábrica" se extendió durante las $24 \mathrm{hs}$ del día incluyendo los fines de semana.
} 


\title{
El espacio de "la producción"
}

Hasta el momento de la recuperación, la organización del trabajo suponía una ordenación espacial según la cual las secciones del proceso productivo se distribuían en los diferentes pisos que componían la planta siendo que a cada piso le correspondía una sección dentro de la cual cada trabajador/a tenía asignado un puesto de trabajo fijo. Una de las modificaciones más significativas desarrolladas a partir de la recuperación fue la ruptura de esta fijación entre actividad y trabajador/a -que en muchos casos se traducía en una asimilación entre un/a trabajador/a y su máquina-, a partir de la rotación de los puestos de trabajo. Así, las/os trabajadoras/es comenzaron a desempeñar diferentes actividades que en muchos casos nunca habían realizado y los puestos pasaron a ser móviles o más precisamente desaparecieron como tales. En relación a este punto es necesario señalar que la rotación de los puestos de trabajo introducida con la recuperación se diferencia de las modalidades de polivalencia impuestas en empresas capitalistas 'tradicionales'. En estas últimas, el objetivo consiste en reducir el costo de la mano de obra a partir de la disminución del número de trabajadoras/es. A diferencia, en este caso la finalidad de la rotación consistía en sustituir aquellos puestos que habían quedado 'vacantes'. En primer lugar fue necesario cubrir tareas de administración y venta pero también actividades más estrictamente ligadas a la producción, sobre todo aquellas que correspondían a puestos de mayor remuneración -como fue el caso de corte o tizado- ${ }^{15}$. Con el tiempo, la rotación se extendió más allá de la 'necesidad' para convertirse en una herramienta que permitió modificar la organización de la producción. Esta modificación tuvo una serie de consecuencias significativas sobre la organización del trabajo que me interesa señalar.

En primer lugar, la rotatividad favoreció la posibilidad de cubrir tareas que momentáneamente quedaban vacantes -como en el caso de licencias o ausencias puntuales- o concentrar el número de personas desempeñando una tarea específica en momentos puntuales ajustando los tiempos y ritmos de trabajo en función de la producción. Pero además este cambio redundó en una serie de beneficios respecto de las condiciones de trabajo, tal como lo expresaba Lorena, una obrera de 35 años que tenía 8 años de antigüedad en la fabrica al momento de la ocupación

\begin{abstract}
"vos estas parada tres horas seguidas, después querés caminar te duelen las rodillas, entonces si rotas, la emoción de hacer rápido, te pasan otro trabajo la emoción de otro laburo... porque vos querés sacar y hacerlo bien. Así rotativo es mejor para mí. Si lo pude llevar a cabo lo hice. Cuando estábamos ahora con la toma, yo le decía a Lidia, "bueno yo hago dos de éstos, y después páseme otra cosa", por ejemplo a plancha, nueve horas paradas con la plancha yo le decía, "no, yo nueve horas no voy a estar parada, lo estuve con la patronal no voy a estar ahora, rotemos" Y con las chicas decíamos, "dos horas cada una", entonces para abrir costura, y dicen "pero todas no sabemos", bueno pero aprendamos, y entonces nos empezamos a rotar la plancha, que es un trabajo horrible. Y más en el verano es horrible. Y bueno nos rotábamos, dos horas, dos horas cada una, entonces sacábamos una cierta cantidad”.
\end{abstract}

El relato de Lorena ilumina el pasaje de una rotatividad 'por la necesidad' a un mecanismo que promovió el desarrollo de mejores condiciones de trabajo, favorecido tanto por el cambio en las

15 Quienes llevaron adelante la recuperación se encontraban en su totalidad empleadas/os como operarias/os o personal de limpieza. No participaron empleados administrativos, de venta ni personal jerárquico (como encargadas/os de sección). Esta situación se repite en la amplia mayoría de las empresas recuperadas, tal como lo reflejan los diferentes relevamientos. A modo de ejemplo, según el informe del Programa Facultad Abierta mencionado previamente, el 69,44\% de las/os trabajadoras/es se encontraban en este grupo. En el caso de esta fábrica, las/os obreros que ocupaban puestos de "corte" o "tizado" -que correspondían a las categorías laborales de mayor calificación- participaron en una menor medida y en varios casos permanecieron poco tiempo luego de iniciada la producción de manera autogestiva. A diferencia de las tareas de ensamblado, en el caso del corte y tizado las actividades permanecieron concentradas en un puesto de trabajo realizado por un trabajador. Estos puestos fueron ocupados por varones que realizaban previamente trabajo de plancha -a vapor- o bien trabajadores que ingresaron a partir de la recuperación -como familiares-. 
posturas (no estar parada durante nueve horas) como por el entusiasmo que genera variar las tareas, "la emoción de otro laburo" según sus palabras. La narración de esta trabajadora nos hace además reparar en otro elemento que merece la pena señalar: la recuperación habilitó a una reflexión colectiva sobre la organización del trabajo en base a la cual fue posible introducir modificaciones sobre la marcha compartiendo las actividades más exigentes en términos corporales como en este caso el trabajo de "plancha".

Ahora bien, como también lo señala Lorena, para poder cambiar de tarea y rotar fue necesario previamente aprender. En este aspecto radica la segunda consecuencia que me interesa destacar. Si por un lado este aprendizaje permitió adquirir capacidades y un mayor manejo del proceso de trabajo, significó también una sobrecarga en términos de "responsabilidades" tal como lo mencionaba Pedro, un obrero de 45 años:

\footnotetext{
"nos sentimos más responsables, más capaces. Y nos tenemos que capacitar en muchas cosas. Antes el tema de la plata estaba a cargo de los patrones, el tema de la administración estaba en las secretarias, la parte de ventas estaban los vendedores, que se arreglen ellos. Pero ahora nos encontramos que todo lo tenemos que hacer nosotros... tenemos que ser vendedores, telefonistas, administrativos, manejar el trabajo, ver que el trabajo salga y que esté en manos nuestras, tener una gran responsabilidad".
}

Así, este aprendizaje conquistado a partir de la recuperación, se extendió más allá de la adquisición de conocimientos relativos a la producción para incluir un amplio abanico de saberes que incluyen cuestiones vinculadas a la administración, comercialización y venta de los productos; la organización interna de la cooperativa; la posibilidad de intervenir en una asamblea; la capacidad de negociar con funcionarios públicos o "pelear" por sus derechos, extendiéndose mucho más allá de "lo productivo". Como nos decía Sandra, una obrera de 48 años, en una charla informal

"Nosotros hacemos asambleas y discutimos, y yo creo que la discusión vale mucho, porque aprendemos mucho, a través de la discusión y todo lo que pueda haber acá. Yo te digo, si tendría que caer de nuevo en una fábrica, no sé si me van a joder tan fácil ya, con lo que pasamos acá. Ya no confiaría en el patrón. Yo aprendí muchas cosas...".

Al mismo tiempo, estos cambios implicaron una sobrecarga de trabajo y la aparición de conflictos internos relacionados con diferencias de actitud de cada una/o con "el trabajo", como lo relataba otra obrera:

\footnotetext{
“yo veo acá que hay responsabilidad sólo en pocos. Y yo soy una de esas que me siento responsable de que salga esto, que salga el otro y que no hay un acompañamiento general de muchos, que vienen y cumplen su horario y se van tranquilos y encima a lo mejor en la asamblea empiezan a cuestionarte todo cuando sos vos la que te estás quedando, sos vos la que estás haciendo, sos vos la que estás tratando y bueno, eso ahora bastante cambió (...) nosotros somos todos trabajadores, pero es un negocio nuestro... Pero lo que más me molesta es por ejemplo que no somos todos los que están pensando en ese trabajo y entonces todo eso te da un poco de bronca..."
}

Las frases citadas corresponden a un fragmento de esta entrevista realizada con Adriana, una obrera de 48 años que antes de la recuperación estaba empleada como oficial calificada y luego pasó a ocuparse de diversas tareas siendo encargada con otras compañeras de la comercialización. Sus palabras expresan con claridad una tensión que observé de manera cotidiana en mis visitas de campo sobre la manera en que se asumía "el trabajo". En este caso, Adriana destaca las diferencias entre quienes se responsabilizan por el trabajo colectivo, lo cual supone para ella un cambio de actitud respecto del pasado, haciéndose cargo "del negocio" más allá de "cumplir con un horario" y asumir los problemas, "estar pensando en ese trabajo".

Un tercer aspecto que me interesa señalar refiere a la desaparición de las categorías ocupacionales previas que se tradujo en un cuestionamiento de las jerarquías salariales. Las antiguas categorías de ocupación indicaban tareas específicas con remuneraciones diferenciales 
en base a determinadas 'calificaciones'. A partir de la recuperación el ingreso dejó de ser un salario para convertirse en una redistribución del ingreso obtenido colectivamente por partes iguales. Las palabras de Carolina, una mujer de 35 años con 7 de antigüedad en la empresa al momento de la recuperación, van en este sentido:

\begin{abstract}
"Una maquinista oficiala calificada... que es alguien más importante, alguien que te maneja un trabajo que uno que te esté barriendo el piso. O sea nadie tiene un cargo más alto que el otro. Vos barrés el piso, vas a ganar igual que yo que estoy en una terminación y soy planchador. O sea que eso con la patronal, el aprendiz gana dos pesos, el calificado gana 15 pesos. Esa es la diferencia con la patronal. Acá no. Acá vos ganas dos pesos, todos ganamos dos pesos. Ganas 10 pesos, ganamos diez pesos. Si no, 30 pesos como pasó, nos llevamos 30 pesos todos. Aunque sobre, pero 30 pesos para todos. Por partes iguales. Porque si no pasaría como cuando está la patronal: que el jefe, el encargado ganan más que el otro y vos te matás laburando y el otro que se pasea ida y vuelta y te miran se llevan 2000 pesos y vos te estás llevando 100 pesos".
\end{abstract}

En el fragmento de esta entrevista, Carolina hace referencia al sentido que adquiere para ella la redistribución de los ingresos que comparte con sus compañeros. Esta resulta no solamente un reparto igualitario de aquello que se produjo colectivamente. Significa también la puesta en cuestión de las categorías salariales que en base a 'calificaciones' reducían los saberes a un aspecto técnico estableciendo jerarquías en los niveles de ingreso. En relación a este punto, resulta interesante tener en cuenta las discusiones que fueron apareciendo cuando los niveles de producción se incrementaban. Estas discusiones centraron en la posibilidad de incorporar nuevas/os trabajadoras/es en pos de intensificar la productividad generando una serie de controversias sobre el porqué y el cómo de esta incorporación. En primer lugar se debatía sobre las limitaciones para incluir personas que, una vez pasado el momento de mayor productividad, resultarían un 'costo' del que habría que hacerse cargo. En segundo lugar, se discutía el status de aquellos que se incorporaban: qué deberes y obligaciones les correspondían en términos de ingresos y participación en la cooperativa. Según mis registros de campo, las personas que se incorporaron fueron formalmente incluidas a la cooperativa como asociadas/os, después de un período "de prueba" y en consecuencia el ingreso que percibieron correspondió al equivalente del colectivo ${ }^{16}$. La discusión se planteó principalmente respecto de la participación y el peso de la palabra en las asambleas más que en relación al nivel de ingreso. Se estableció así una distinción entre quienes habían "puesto el cuerpo para salvar la fuente de trabajo" y las/os "nuevas/os", definiendo un principio de legitimidad adquirido en "la lucha". En tercer lugar, las discusiones versaron sobre quiénes debían ser incorporados: trabajadoras/es desocupadas/os principalmente aquellos que se habían solidarizado con "la lucha"-, familiares en situaciones similares o bien trabajadoras/es "de oficio", principalmente ex empleadas/os de la empresa. El carácter que adquirió esta discusión puso en evidencia una tensión que comenzó a desarrollarse de manera creciente y podemos sintetizar entre "compromiso con la lucha" y "compromiso con la producción". Mientras que para algunas/os comprometerse con la recuperación suponía asumir mayor "responsabilidad con el trabajo", en los términos en que Adriana lo definía en el fragmento citado más arriba, para otras/os este compromiso requería devolver la solidaridad recibida, acompañar "la lucha" de otros colectivos o asumir una actitud combativa como

\footnotetext{
${ }^{16}$ Las cooperativas de trabajo conformadas a partir de empresas recuperadas deben regirse por la legislación vigente, correspondiente a la ley 20337 del año 1973, que estipula la incorporación de las/los trabajadoras/es deben como asociadas/os. El Instituto Nacional de Asociativismo y Economía Social (INAES) establece en estos casos la posibilidad de un período de prueba de seis meses previo. La modificación de esta legislación en pos de una nueva ley que contemple la realidad actual de las cooperativas de trabajo constituye una de las principales demandas de algunas organizaciones que nuclean estos colectivos. Como lo han señalado diferentes estudios la incorporación de nuevos trabajadores en las empresas recuperadas es uno de los principales problemas a los que se enfrentan estas experiencias. Estos estudios señalan que en algunos casos se han introducido pagos diferenciales basados en la antigüedad en la fábrica (sobre todo en base a una distinción entre "viejos" y "nuevos" asociados) o las responsabilidades asumidas, desarrollando lo que algunos autores han caracterizado como formas de "desigualación emergente" (Rebón y Salgado, 2009).
} 
organización obrera. En definitiva, esta tensión puso en escena una discusión creciente sobre el sentido mismo de la recuperación y la manera en que se definían quienes de ella participaban.

En síntesis, la rotatividad de los puestos de trabajo y las consecuencias que este cambio trajo en términos de la organización de la producción ilustra el modo en que a partir de la recuperación la política fue redefiniendo el sentido del trabajo. En sentido inverso, en el próximo apartado me propongo desarrollar la manera en que en el marco de este proceso de demanda y organización colectiva el lenguaje del trabajo (productivo) permeó las acciones de protesta desarrolladas, modelando sus formatos, ritmos y dinámicas.

\section{El espacio de "la lucha"}

En abril de 2003 las obreras y obreros fueron desalojados de la fábrica mediante un operativo policial de gran envergadura que finalizó en una fuerte represión y el vallado de la planta ${ }^{17}$. Sin posibilidades de ingresar a la fábrica, las/os trabajadoras/es montaron frente a sus puertas un acampe que se prolongó durante los nueve meses siguientes. El acampe tenía el objetivo de custodiar la planta ante la posibilidad del retiro de máquinas u otros bienes y continuar la "lucha" manteniendo la demanda por "la fuente de trabajo". Para ello se levantó una carpa ubicada en una plaza a pocos metros de la planta en la que las/os trabajadoras/es, acompañados por militantes de organizaciones sociales y políticas, permanecían durante las 24 horas del día. Transcribo a continuación un fragmento de mis registros de campo durante el acampe que reconstruye una mañana en la carpa.

Minutos antes de las 10 de la mañana fueron llegando las/os últimas/os. Cumpliendo con el horario de llegada pautado, una/o a una/o firmaron el cuaderno de presentes de la misma forma que lo hacían cuando estaban adentro de la fábrica. Quienes pasaron la noche en la carpa "cubriendo su guardia", hacía ya algunas horas que estaban despiertos. Mientras esperaban el comienzo de la asamblea, los recién llegados acomodaron sus cosas en un pequeño estante donado por un vecino del barrio. Como habitualmente lo hacían en la fábrica, dejaron en él la ropa con la que llegaron a la carpa y se pusieron el guardapolvo celeste, convertido en una marca de "su lucha". Luisa, una obrera de 32 años, repartió a cada una/o un papel con el turno y la dirección del Banco Ciudad al que debían acercarse a cobrar el subsidio que consiguieron a partir de las negociaciones en la carpa con funcionarios de "acción social". Esa mañana la agenda del día incluía la visita de un legislador y una reunión con un representante de la defensoría del pueblo. La pava estaba lista y el mate preparándose. Beatriz, Inés y Rosana, las encargadas de cocinar para ese mediodía, salieron a conseguir huevos y verduras que faltaban para hacer torrejas con el arroz que todavía queda de "los bolsones" de alimentos recibidos. Al fondo Irma y Margarita, tejían batitas de bebé y medias de lana, mientras comentaban con algunas de sus compañeras cuándo les toca la próxima guardia. Me senté cerca de ellas. Irma me contó que estaba tejiendo para vender en el barrio y que con eso sacaba algo de plata. Al rato Diana se aproximó para comentarles que un cliente se contactó para ofrecerles "un trabajito". Explicó de qué se trataba y les dijo que estaban pensando en repartirlo para que cada uno haga una parte y "pueda quedarse con algo".

Como queda reflejado en este fragmento de mis notas de campo, durante el acampe se recrearon los horarios de llegada y salida, las guardias, los controles internos, la distribución de tareas y los modos de llevarlos adelante. De esta manera, aunque con pequeñas modificaciones en los horarios, el acampe fue organizado siguiendo las temporalidades y dinámicas del día a día en la fábrica. A mediados de 2004, meses después del reingreso a la planta, mantuve una

\footnotetext{
${ }^{17}$ Como mencionamos previamente este fue el tercer desalojo al que hicieron frente desde el inicio de la ocupación. En los dos primeros, en marzo y noviembre de 2002, las trabajadoras y trabajadores habían conseguido permanecer o reingresar rápidamente en la fábrica. En este caso el ingreso a la planta se produjo en diciembre de 2003, luego de un prolongado proceso de movilización que finalizó con la sanción de una ley de expropiación.
} 
conversación con Ana, una trabajadora de 32 años quien había participado de la ocupación y el comienzo de la gestión obrera hasta que tuvo lugar el desalojo. En este fragmento que transcribo a continuación, Ana relataba el momento en que había decidido "volver a trabajar" mientras sus compañeras mantenían el acampe instalado frente a la fábrica:

MI: ¿Y en la carpa sí estuviste o no?

A: Si, después volví, tenía 2 meses la nena, a los 3 meses volví, me separé y bueno, me separé y me puse a pensar y bueno, tengo que volver a trabajar y...

MI: ¿Tu marido trabajaba?

A: Trabajo fijo no tenía, y bueno y entonces yo agarré y bue... tengo que volver a trabajar. Querer volver acá no quería viste? no sé, no le veía otra cosa por el momento, que si veía otra cosa mejor me iría en otro lado, pero lo pensé voy a volver, y cuando volví a los 3 meses del quilombo que hubo [en referencia al momento del desalojo y la represión] estaba la carpa, me presenté y alguna me decía bueno, si vos volvés, si decís que vas a volver quedate, pero no vaya a ser cosa que te vuelvas a ir. Bueno, yo vengo y me quedo ((risas)). "Tenés que hacer las guardias, tenés que quedarte a la noche, tenés que hacer esto" y bueno.

Las palabras de Ana reflejan con claridad este modo de significar y vivir el acampe como un espacio "de trabajo" en varios sentidos. Por un lado, al igual que la fábrica, la carpa es un espacio al que puede "presentarse" a trabajar. En esta línea, la carpa relocalizó el espacio relacional de la fábrica sobre una geografía que pese a carecer de los marcadores referenciales propios de un espacio laboral fabril, era igualmente significado como el 'lugar de trabajo'. En consecuencia, permanecer en la carpa supuso cumplir ciertas obligaciones como hacer las guardias, pasar la noche, realizar determinadas actividades que en este caso, en lugar de tareas productivas correspondían principalmente a prácticas "de lucha": movilizarse, cortar la calle, asistir a reuniones de negociación con funcionarios, garantizar la comida, etc. De esta manera, el espacio de la carpa era para una visitante externa un ámbito sumamente regulado dentro del cual las personas que participaban tenían asignadas tareas específicas que debía cumplir. Así, mientras que desde el 18 de diciembre la recuperación había implicado un proceso por el que el "lugar de trabajo" se convertía al mismo tiempo en un "espacio de lucha" en el acampe esta relación fue invertida haciendo del "espacio de lucha" un "lugar de trabajo". Desde un punto de vista analítico, me interesa destacar el modo en que la recuperación tomó la forma de una acción política anclada en el trabajo productivo.

Para ilustrar este punto, me detendré en una acción de protesta desarrollada particularmente durante este período bajo el nombre de maquinazo. Estas acciones estuvieron dirigidas expresamente a lograr la sanción de un proyecto de ley de expropiación presentado en la Legislatura de la Ciudad de Buenos Aires ${ }^{18}$ y consistían en ocupar la vía pública instalando máquinas de coser de uso familiar que habían sido donadas por vecinos del barrio, militantes de asambleas y de organizaciones sociales. Durante los maquinazos las/os trabajadoras/es, principalmente las mujeres, se disponían a coser por el lapso de algunas horas vestidas con sus guardapolvos celestes. Aunque en la mayoría de los casos tuvieron lugar frente a la fábrica vallada, en algunas ocasiones se realizaron ante organismos públicos como la Casa de Gobierno de la Ciudad de Buenos Aires o la Legislatura local. El énfasis de esta acción de protesta estaba puesto en mostrarse trabajando, dando pruebas de su capacidad para realizar este oficio -como el manejo de las herramientas- y de su voluntad de trabajo, de seguir siendo trabajadoras/es. En este sentido, es posible pensar al maquinazo como un corte de calle, una modalidad de acción compartida con otros -principalmente "los desocupados"- que en el marco de este proceso de

\footnotetext{
${ }^{18}$ A mediados de mayo el legislador Rodríguez elaboró un proyecto de ley con características similares a las leyes sancionadas durante 2002 frente a las empresas recuperadas. Era el segundo proyecto de ley que se presentaba en la legislatura en nombre de esta recuperada. El primero, rechazado en octubre de 2002, planteaba la expropiación de la empresa y la transferencia de la propiedad al Estado de la Ciudad de Buenos Aires que sería el encargado de mantener el funcionamiento de la misma bajo el control y la administración de los trabajadores.
} 
demanda adquirió características singulares, diferenciándose así de estos últimos. En este caso, el corte de la calle se realizaba trabajando. Más aún, se cortaba la calle para poder trabajar. El uso de ropa de trabajo que las/los identificaba como trabajadoras/es de un determinado oficio realzaba esta demanda. En este caso, el guardapolvo celeste expresaba una distinción del colectivo de trabajo marcando así la capacidad de desarrollar una actividad particular: la confección. Aunque la realización de maquinazos fue una práctica singular de este proceso, las/os trabajadoras/es de otras recuperadas llevaron adelante acciones con características similares, como la exposición de herramientas de trabajo en la vía pública, la realización de muestras con stands en las que se exponían sus productos o la distribución de estos últimos en ámbitos como la legislatura o el congreso ante el posible tratamiento de una ley.

A mi entender, comprender la significación de estas prácticas requiere prestar atención a las principales características de las políticas públicas en materia de empleo desarrolladas en los años previos en Argentina (Lo Vuolo y Barbeito, 1998; Lindemboin y Danani, 2003; Grassi, 2003). Me refiero específicamente a las medidas implementadas durante los años '90, financiadas por organismos de crédito internacional y destinadas a paliar "los efectos colaterales" del ajuste neoliberal (Cross y Freytes Frey, 2009). Conocidas como Programas de Empleo Transitorio (PET), estas políticas siguieron la lógica del modelo de workfare promovido por el Banco Mundial que orientó las intervenciones hacia la asignación de una ayuda financiera cuyo monto no debía ser más bajo que el salario mínimo, exigiendo a los beneficiarios una contrapartida en trabajo (Gautié, 2002) ${ }^{19}$. En este contexto, los planes se constituyeron en la referencia a un modo de trabajo que no se considera genuino -incluso para las organizaciones que demandaban cupos- (Fernández Álvarez y Manzano, 2007). Fue frente a estas políticas que las empresas recuperadas sostuvieron sus reivindicaciones exigiendo al Estado otro modo de intervención que garantizara un "trabajo digno y genuino" manteniendo su "fuente de trabajo". En este sentido, otros estudios han observado modos similares de significar la participación en procesos de demanda por trabajo como fue el caso de las organizaciones de desocupados donde la presencia cotidiana de beneficiarios de estos "planes" fue definida en términos semejantes (Quiros, 2006; Manzano, 2009; Cross, 2010; Rius, 2011). Al igual que la descripción previa del acampe, en estos casos las personas referían a su participación en los movimientos como "un trabajo" y las actividades realizadas fueron organizadas, reguladas y definidas reproduciendo un esquema propio del mundo laboral fabril: cumplimiento de horarios, distribución de responsabilidades, etc. Lo interesante en este caso es justamente el modo en que al tiempo que la participación en estos procesos de demanda fue definido y organizado como un trabajo, las acciones de protesta cobraron formatos, sentidos, prácticas y discursos propios del mundo laboral, en particular del espacio fabril. En una palabra, articularon un lenguaje de la protesta, siguiendo la categoría de Roseberry (1994), que apelando al trabajo como condición de vida posibilitó no sólo que esta demanda fuera escuchada sino también conquistada.

\section{Palabras finales}

\footnotetext{
${ }^{19}$ A partir del año 1993 se pusieron en marcha una variedad de programas bajo esta lógica dentro de los cuales el más importante fue el "Trabajar" implementado dos años después. Las personas eran empleadas por un período mayor a tres meses (el tiempo límite del período de prueba para las empresas privadas a partir de la ley de empleo de 1991) y menos de doce y recibían una contrapartida económica, definida como "ayuda no remunerativa" que iba entre 150 y 300 pesos (en aquel momento equivalente a 300 dólares). Con muy pocas modificaciones, en los años siguientes, se elaboran programas semejantes bajo el nombre de Trabajar II y III y en enero de 2002 los PET se unificaron en el Programa Jefas y Jefes de Hogar para Desocupados implementado en el marco de la declaración de la emergencia social, económica y administrativa Dirigido a Jefes y Jefas de hogar desocupados con hijos menores a cargo, quienes reciben una suma mensual de 150 pesos a cambio de participar en actividades de capacitación, comunitarias o productivas. Con un desarrollo más importante en términos de población objetivo, este último alcanzó en el primer año los 2.000.000 de beneficiarios.
} 
Uno de los aportes más significativos de la antropología al estudio de los procesos de movilización social radica en desplazar la mirada de los momentos épicos y visibles para ponderar el estudio de las prácticas cotidianas (Alvarez, Escobar y Dagnino, 1998; Edelman, 2001). El aporte de James Scott, paradójicamente un politólogo, constituye sin duda uno de los planteos más influyentes. Su trabajo discute con los estudios sobre movimientos sociales y acción colectiva que partiendo del análisis de procesos desarrollados en regímenes democráticos invisibilizaron las prácticas de resistencia que de manera cotidiana se desarrollaban en contextos opresivos. En estos contextos, siguiendo al autor, la expresión "pública" del descontento no era posible y por lo tanto debía darse de manera "oculta", expresándose en canciones, chistes, burlas, etc. (Scott, 1987; 2000). Así, tendió a establecerse una dicotomía que contrapuso las expresiones públicas de confrontación abierta, a las formas ocultas de resistencia cotidiana. El trabajo de J. Scott fue objeto de numerosas críticas que cuestionaron la visión romántica de su planteo sobre lo que el autor llama "los dominados", así como la mirada dicotómica desde la que analiza sus prácticas en contraposición a aquellas de "los dominantes", en las que ambos grupos se contraponen y homogeneízan a su interior (Ortner, 1995; Gledhill, 2000). Me interesa en particular aquí retomar la discusión que propone el antropólogo M. Guttman (1993) al afirmar que uno de los principales problemas de la propuesta de Scott reside en situar las formas de resistencia cotidiana en el espacio de lo "oculto" y por lo tanto de la "infrapolitica". Creo que en este sentido, el análisis del proceso de recuperación y la manera en que en este marco se articularon "política" y "trabajo" puede aportar algunos elementos para avanzar en esta reflexión.

Como decía al comienzo de este artículo, las empresas recuperadas fueron objeto de una importante producción académica y militante a la que aportaron no solo autores provenientes de la Argentina sino de otras regiones del mundo. Parte de esta literatura interpeló a estas experiencias por su carácter alternativo e insurgente emparentando estas acciones con otros procesos que a nivel regional parecían desafiar la globalización y el neoliberalismo. Estos estudios dieron proyección mundial a las empresas recuperadas que fueron reconocidas en países de la región como Brasil o Venezuela donde se desarrollaron experiencias similares, o incluso más allá de América Latina interpelando la realidad de otros países como Estados Unidos, España o Francia. Se ponderó así una mirada que tendió a preguntarse por la radicalidad (o no) de estas acciones, analizando ya sea su potencial político o bien su capacidad de desarrollar modelos alternativos de organización del trabajo. En consecuencia, los estudios abordaron las prácticas de movilización iluminando los momentos épicos y públicos o bien contemplaron los procesos de organización del trabajo haciendo foco en las prácticas cotidianas.

Entiendo que uno de los aportes más sustantivos que las empresas recuperadas permiten hacer radica justamente en la manera en que trabajo y política se articularon en la cotidianeidad de estas experiencias. Lo que supone a mi criterio pensar a las empresas recuperadas como prácticas cotidianas de "lucha" y "trabajo" que permitieron desarrollar un proceso innovador de demanda. Se trató de un hacer del día a día, una construcción cotidiana en la que sus protagonistas reinventaron desde su experiencia de vida modos renovados de hacer política y trabajo. Y esta cotidianeidad, retomando el planteo de Guttman, no tuvo lugar en un plano "oculto" sino principalmente en una resistencia cotidiana basada en la exposición pública de prácticas de lucha ancladas en el trabajo.

\section{Bibliografía citada}

ALVAREZ, S.; ESCOBAR, A.; DAGNINO, E. -eds- (1998) Cultures of Politics/Politics of Culture: Revisioning Latin American Social Movements. Boulder. Westview Press.

DAVOLOS, P. y PERELMAN, L. (2005) "Respuestas al neoliberalismo en Argentina". Política y cultura. n.24, Universidad Autónoma de México, Unidad Xochimilco. México DF. pp. 207-229. 
GRACIA, A. y CAVALIERE, S. (2007) "Repertorios en Fábrica. La experiencia de recuperación fabril en Argentina, 2000-2006." Estudios Sociológicos Vol. XXV, núm. 73, enero-abril. El Colegio de México. México DF pp. 155-18

CROSS, C. (2010) “Ves otras personas en nosotros mismos': Experiencias de vinculación en organizaciones territoriales de Buenos Aires" Cuadernos de Antropología Social, No 31. Instituto de Ciencias Antropológicas, Facultad de Filosofía y Letras, Universidad de Buenos Aires, Ciudad de Buenos Aires, pp. 55-74.

CROSS, C. y FREYTES FREY, A. (2009) "Políticas sociales como límite y como herramienta: Reflexiones a partir de experiencias de gestión de dirigentes y referentes piqueteros/as en el período 2001-2007", El Príncipe, № 1, Asociación de Politólogos Bonaerense, La Plata pp. 7598.

EDELMAN, M. (2001) "Social Movements: changing paradigms and forms of politics", Annual Review of Anthropology, Vol 30. Annual Review. Palo Alto, California. pp: 285-317

FAJN, G. y REBON, J. (2005), "El taller ¿sin cronómetro? Apuntes acerca de las empresas recuperadas", Herramienta, № 28, marzo, Ediciones Herramienta, Buenos Aires, pp. 47-66.

FAJN, G. (2003), Fábricas y empresas recuperadas. Protesta social, autogestión y rupturas en la subjetividad, Centro Cultural de la Cooperación. Buenos Aires.

FAVARO, O. y AIZICSON, F. (2003), "La resistencia obrera en Zanón, Neuquén”, Realidad Económica, $\mathrm{N}^{\mathrm{o}}$ 197, julio-agosto. Instituto Argentino para el Desarrollo Económico. Buenos Aires. pp. 24-39.

FERNÁNDEZ ALVAREZ, MI (2007). "De la recuperación como acción a la recuperación como proceso: prácticas de movilización social y acciones estatales en torno a las recuperaciones de fábricas", Cuadernos de Antropología Social, № 25, Instituto de Ciencias Antropológicas, Facultad de Filosofía y Letras, Universidad de Buenos Aires, Ciudad de Buenos Aires, pp 89-110.

FERNÁNDEZ ÁLVAREZ, M. I. y Manzano, V. (2007): "Desempleo, acción estatal y movilización social en Argentina", Revista Política y Cultura. № 27.Universidad Autónoma de México, Unidad Xochimilco México D. F. pp: 143-166.

GAUTIE, J. (2002), "De la invention du chômage á sa deconstruction", Geneses, No 46, Centre Nationale du Livre y Centre national de la recherche scientifique, Paris. pp. 60-76.

GLEDHILL, J. (2000) El poder y sus disfraces. Perspectivas antropológicas de la política. Ediciones Bellaterra. Barcelona.

GRASSI, E. (2003), Políticas y problemas sociales en la sociedad neoliberal. La otra década infame (I). Espacio Editorial, Buenos Aires.

GUTTMAN, M (1993) "Rituals of resistence: A critique of the theory of every forms of resistence". Latin American Perspectives. Vol 20 N2, SAGE. pp. 74-92.

Hudson, J. P. (2007) "Empresas autogestionadas y producción de subjetividad" VII Jornadas de Sociología de la UBA, Buenos Aires.

LINDENBOIM, J. y DANANI, C. (2003) Entre el trabajo y la politica. Las reformas de las politicas sociales argentinas en perspectiva comparada, Biblos. Buenos Aires.

LO VUOLO, R. y BARBEITO, A. (1998), La nueva oscuridad de la política social. Del estado populista al neoconservador, Ciepp-Miño y Dávila, Buenos Aires

MAGNANI, E. (2003) El cambio silencioso. Empresas y fábricas recuperadas por los trabajadores en la Argentina, Prometeo, Buenos Aires. 
MANZANO, V. (2009) "Un barrio, diferentes grupos: acerca de dinámicas políticas locales en el distrito de La Matanza”. En: Grimson, A.; Ferraudi Curto, C. y Segura, R. (comps.) La vida politica en los barrios populares de Buenos aires. Prometeo, Buenos Aires. pp: 267-291.

MARTINEZ, J. (2002) "Fábricas ocupadas y gestión obrera directa. Apuntes para una reflexión histórica y teórica." Revista La Chispa. Rosario.

NASH, J. (2006) Visiones Mayas. El problema de la autonomía en la era de la globalización. Antropofagia. Buenos Aires.

NASH, J. - ed- (2005) "Introduction" In: Social Movements: An Anthropological Reader, New York, Blackwell

QUIRÓS, J. (2006). Cruzando la Sarmiento. Una etnografia sobre piqueteros en la trama social del sur del Gran Buenos Aires. Antropofagia. Buenos Aires.

ORTNER, SH. (1995) "Resistance and the problem of ethnographic refusal". Comparative studies in society and history, Vol. 37 N1, University of Michigan, pp.173-193.

PALOMINO, H. (2003) "Las experiencias actuales de autogestión en Argentina". Revista Nueva Sociedad No 184. Fundación Friedrich Ebert (FES), pp. 115-128.

PETRAS, J. y VETMEYER, H. (2002) “Autogerenciamiento de trabajadores en una perspectiva historica" En: Carpintero, E. y Hernandez, M. Produciendo Realidad Las empresas comunitarias. Buenos Aires, Topia.

REBÓN, J. (2007) La empresa de la autonomía. Trabajadores recuperando la producción, Ediciones Picasso/ Colectivo Ediciones, Buenos Aires.

REBÓN, J. y SALGADO, R. (2009), "Desafíos emergentes de las empresas recuperadas: de la imposibilidad teórica a la práctica de la posibilidad", Julio Neffa, Enrique De la Garza Toledo y Leticia Muñiz Terra (comp.) Trabajo, empleo, calificaciones profesionales, relaciones de trabajo e identidades laborales Vol. II CLACSO/CEIL-PIETTE/Trabajo y Sociedad, Buenos Aires, pp 29-58

ROFMAN, A., SLUTZKY, D. Y DI LORETO, M. (2003) "Experiencias autogestionarias en un marco de crisis económico-social inédita: las empresas recuradas" VI Congreso Nacional de Estudios del Trabajo, Buenos Aires, 13 al 16 de agosto.

RIUS, P. (2011) "Del Movimiento de Trabajadores Desocupados a la cooperativa social. Trabajo y formas de militancia en la economía social" En: Revista Trabajo y Sociedad, No 17, vol. XV, Invierno, Departamento de Sociología de la Universidad Nacional de Santiago del Estero. pp. 265-283.

ROSEBERRY, W. (1994), "Hegemony and the Language of Contention", En Joseph Gilbert y Daniel Nugent (eds.) Everyday Forms of State Formation. Revolution and ther Negortiation of Rule in Modern Mexico. Duke University Press, Dirham and London, pp 355 - 366.

RUGGERI, A. -comp.- (2009). Las empresas recuperadas. Autogestión obrera en Argentina y América Latina. Facultad de Filosofía y Letras, Universidad de Buenos Aires, Ciudad de Buenos Aires.

SCOTT, J. (1987) "Resistance without protest and without organization: Peasant opposition to the Islamic Zacat and the Christian Tithe" Comparative studies in society and history Vol 29 University of Michigan pp: 417-452”.

SCOTT, J. (2000) Los dominados y el arte de la resistencia. Discursos ocultos. Ediciones Era. México D.F.

TRINCHERO, H. (2007) "Economía política de la exclusión. Para una crítica desde la experiencia de las empresas recuperadas por sus trabajadores (ERT)" Cuadernos de 
Antropología Social. N26 Instituto de Ciencias Antropológicas, Facultad de Filosofía y Letras, Universidad de Buenos Aires, Ciudad de Buenos Aires. pp. 41-67. 